\title{
Intervención educativa en mujeres fértiles para elevar el conocimiento del flujo vaginal
}

Educational intervention in fertile women to raise awareness of vaginal discharge

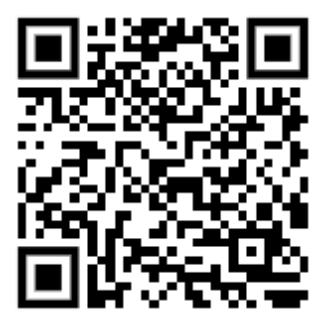

RECIBIDO

$12 / 02 / 2019$

\author{
${ }^{1}$ Dr. Raydel Pérez Castillo \\ Centro Provincial de Medicina Deportiva, Las Tunas, Cuba \\ raydelperezc@nauta.cu \\ https://orcid.org/0000-0001-9454-5375 \\ ${ }^{2}$ Dra Yoanne Cordero González \\ Policlínico Aquiles Espinosa, Las Tunas, Cuba \\ yoacordero@ltu.sld.cu \\ https://orcid.org/0000-0003-0971-458X
}

CORREGIDO

ACEPTADO

9/03/2019

$18 / 03 / 2019$

\section{RESUMEN}

Introducción: El flujo vaginal constituye una de las variante sindrómica más frecuentes entre las patologías ginecológicas y la causa más frecuente de consulta ginecológica en cualquier edad. Se pretende determinar el efecto de una intervención educativa en el conocimiento de los factores predisponentes al flujo vaginal y su repercusión sexualreproductiva.

Método: Se realizó un cuasi-experimento educativo con diseño antesdespués en 23 mujeres en edad fértil de un consultorio médico. Se analizó las variables edad, primeras relaciones sexuales, conocimiento sobre los factores $y$ efectos sexuales del flujo vaginal por una herramienta semi-estructurada previamente validada. La intervención se estructuró en talleres, distribuidos en sesiones de tres horas por 12 semanas. Los resultados de la intervención antes y después se compararon por pruebas inferenciales no paramétrica con el software

${ }^{1}$ Médico especialista en Medicina Familiar, profesor Instructor, Centro Provincial de Medicina del Deporte, Las Tunas, Cuba.

${ }^{2}$ Médico especialista en Medicina Familiar, profesora Asistente, Policlínico Aquiles Espinosa, Las Tunas, Cuba.
Resultados: La edad media y primeras relaciones sexuales fueron de 28 y 15 años respectivamente, con niveles de escolaridad medio y medio-superior. El conocimiento grupal pre-intervención tuvo una mediana de 46.00 puntos, posterior al estudio la misma fue de 78.00 con un nivel de significación estadística $(p<0.05)$.

Conclusión: La intervención educativa basada en la participación contribuye a la adquisición de nuevos conocimientos acerca de la influencia del flujo vaginal en la salud sexual y reproductiva de la mujer. 
PALABRAS CLAVE: salud sexual y reproductiva; microbiota; disbiosis; educación compensatoria.

\section{ABSTRACT}

Objectives: Vaginal discharge is one of the most common syndromic variants among gynecological pathologies and the most frequent cause of gynecological consultation at any age. The aim is to determine the effect of an educational intervention on the knowledge of predisposing factors to vaginal discharge and its sexual-reproductive repercussion.

Objectives: An educational quasi-experiment with a before-after design was carried out in 23 women of childbearing age in a doctor's office. We analyzed the variables age, first sexual relations, knowledge about the sexual factors and effects of vaginal discharge by a semi-structured tool previously validated. The intervention was structured in workshops, distributed in sessions of three hours for 12 weeks. The results of the before and after intervention were compared by nonparametric inferential tests with the Infostat / L software.

Results: The average age and first sexual relations were 28 and 15 years respectively, with average and upper-middle school levels. The pre-intervention group knowledge had a median of 46.00 points, after the study it was 78.00 with a level of statistical significance $(p<0.05)$.

Conclusion: A precocious start of sexual relations exists in women in fertile age. You associate her you witness her of vaginal flow for ignorance of the risk factors and his repercussion in the sexual and reproductive health. The educational intervention based in participation contributes to the acquisition of new knowledge.

KEYWORDS: sexual and reproductive; microbiota, dysbiosis; remedial teaching.

\section{INTRODUCCIÓN}

El flujo vaginal constituye una de las variante sindrómica más frecuentes entre las patologías ginecológicas y la causa más frecuente de consulta ginecológica en cualquier edad (1). Las féminas generalmente tienen como concepto que aparece solo en situaciones de infecciones de transmisión sexual.

Durante la evaluación de los indicadores de salud materna en el primer semestre de 2018 de un Consultorio Médico se diagnosticó que el síndrome de flujo vaginal aparece en una cada tres mujeres al concebir su embarazo.

Las principales insuficiencias estuvieron dirigidas al desconocimiento de las causas del flujo vaginal y las consecuencias de este sobre la sexualidad y reproducción.

Ante la anterior situación se tiene como objetivo determinar el efecto de una intervención educativa en el conocimiento de los factores predisponentes al flujo vaginal y su repercusión sexual-reproductiva en un grupo de mujeres en edad fértil. 


\section{MATERIALES Y MÉTODOS}

Se diseñó y aplicó un estudio cuasiexperimental educativo. La población estuvo constituida por 213 mujeres y una muestra de 23 toda en edad fértil; atendidas por un consultorio médico del Policlínico Aquiles Espinosa en Las Tunas, Cuba en el periodo septiembre a diciembre de 2018. Se estableció como criterio de inclusión haber padecido cuadro de flujo vaginal, aceptar voluntariamente la participación en la investigación, edad superior a los 18 años y permanencia en el área durante la fase interventiva de la investigación.

El conocimiento fue evaluado empleando dos encuestas previamente validadas por método Delphi de 15 preguntas cada una. En la etapa pre-intervención solo 10 tuvieron carácter evaluativo pues cinco preguntas estaban destinas a variables epidemiológicos (edad, primeras relaciones sexuales, rechazo de una pareja por presencia del flujo, escolaridad, orientación anterior del tema) por cada pregunta se otorga un valor de 10 puntos, el resultado final se obtuvo de la adición y sustracción de las respuestas efectuadas. El nivel de conocimiento del flujo vaginal se estandarizó como: a) mal: $\leq 59$ puntos; b) regular: $60-75$ puntos; c) bien: 76 89 y d) muy bien: $\geq 90$ puntos.

La intervención educativa consistió en 36 horas de talleres, distribuidas en una sesión semanal de 3 horas durante 12 semanas, en los cuales, luego de la exposición de un contenido conceptualizado se procedía a la reflexión de cada tema.

Los contenidos temáticos fueron: características normales de la vagina, definición y variantes del flujo vaginal, medidas para la prevención, efecto sobre la reproducción y el disfrute sexual, violación de los derechos sexuales.

En el diseño de la investigación se usó métodos teóricos (análisis-síntesis, inducción-deducción), empíricos (encuesta, la revisión documental) con carácter anónimo para diagnosticar las insuficiencias en el conocimiento antes y después de la intervención; además de método estadísticos ( medidas descriptivas de tendencia central y dispersión, comparación por prueba inferencial de Normalidad S-Wilks modificada y cálculos de inferencia por prueba Wilcoxon) procesados por el software InfoStat/L.

El estudio se realizó en correspondencia con lo establecido en la Declaración de Helsinki, y por las regulaciones estatales sobre los principios de la Bioética. En virtud de que se trató de un estudio de intervención sobre la cognición, la investigación fue considerada con riesgo mínimo.

\section{MARCO TEÓRICO}

En la práctica diaria las principales causas de consulta ginecológica son las leucorreas o flujo y el prurito vaginal, cuyo tratamiento se basa principalmente en las características clínicas, siendo catalogadas en forma arbitraria como vaginosis o vaginitis sin llegar a considerar su etiología. (2)

La vagina es una estructura fibromuscular, que cuenta con tres capas histológicas: mucosa, muscular y adventicia. En la vagina se albergan microorganismos que generan un estatus fisiológico, el cual es importante para el bienestar reproductivo del huésped. La composición de la microbiota vaginal es dinámica y responde a los distintos estados hormonales que atraviesa la mujer en su vida reproductiva. $(3,4)$

En la vagina existe normalmente un contenido que está formado por la mezcla de varios elementos: secreción de las glándulas mucosas de endocérvix, células descamadas de las capas superficiales de la pared y el exocérvix y secreción de las glándulas vestibulares de caracter escaso, blanquecino, homogéneo, espeso, untuoso y de olor alcalino situada en el fondo de saco vaginal. $(5,6)$. El medio vaginal es ácido a causa del ácido láctico que contiene; el proceso de formación de este sirve de alimento a un 
germen saprófito denominado lactobacilo de Döderlein. (5).

La presencia de ácido láctico tiene un papel importante;en ocasiones las concentraciones de lactobacilos vaginales se modifican, produciéndose una disbiosis de la microbiota vaginal, caracterizada por baja presencia de los mismos y sobrecrecimiento de gérmenes anaerobios, la más común se presenta como vaginosis bacteriana. (4)

Desdel punto de vista conceptual el síndrome de flujo vaginal o leucorrea es toda perdida no sanguinolenta que provenga del aparato genital femenino.

En algunas mujeres puede referirse una "mucorrea fisiológica" alrededor de la ovulación, de pocos días de evolución y asintomática, como expresión del pico estrogénico preovulatorio. (5) Por otra parte el flujo vaginal patológico es el estado de desequilibrio o alteración de la flora vaginal que se produce cuando hay una disminución de lactobacilos y una proliferación de agentes infecciosos tanto de bacterias como hongos y parásitos e incluso la aparición de virus" al decir de Perea (7) y Castillo Pacheco. (1)

Se origina por diferentes agentes etiológicos, siendo más frecuente en mujeres en edad fértil sexualmente activa.

Aproximadamente la tercera parte de estas tienen síndrome de flujo vaginal por una o más infecciones, tumores ginecológicos, afecciones hormonales y embarazo. $(9,4,10)$ Las tres infecciones más comunes asociadas al síndrome de flujo vaginal son la trichomoniasis (15-20\%), la vaginosis bacteriana $(40-50 \%)$, y la candidiasis (20$25 \%$ ), en menor medida se presentan la gonorrea y la clamidiasis. $(11,12)$

Existen una serie de factores que influyen en la variación de la microbiota vaginal como son la conducta sexual relacionada con múltiples parejas sexuales, la falta de utilización del condón, el grupo étnico, los trastornos en las hormonas sexuales, el estrés psicosocial, la aplicación de productos de higiene femenina, las duchas vaginales, la alcalinidad transitoria del medio por la menstruación o el semen, uso de antibióticos, tabaquismo y el embarazo. $(3,4,13)$ Relacionado con estos María et al. (14) consideraron el nivel de instrucción especialmente el analfabetismo así como el uso de anticonceptivos como otras situaciones influyentes. El síndrome de flujo vaginal al decir de Estruch Rancaño (15) y citado por Vidal Borrás et col (16) representa en el mundo un serio problema no solo en términos de salud, sino también, en el ámbito económico y social. Su control es decisivo para mejorar la salud sexual y reproductiva al representar uno de los grandes desafíos de la salud pública contemporánea según los estudios de Abril Matute et col. (17). La alteración en la microbiota vaginal puede desencadenar parto pretérmino, infecciones maternas o neonatales, bajo peso al nacer, enfermedad pélvica inflamatoria e infertilidad. Estas situaciones constituyen una causa importante de gasto público en el área de la salud y en el ámbito personal; en el aspecto psicológico, se presenta un desgaste que influye en sus relaciones interpersonales, laborales y conyugales en detrimento de su calidad de vida sexual y reproductiva. El pleno disfrute de la actividad sexual se resquebraja por el dolor durante el coito, el ardor vaginal, la inseguridad o la vergüenza al rechazo por la pareja sexual.

\section{RESULTADOS}

Se estudió 23 mujeres con edad biológica media de $28.6 \pm 6.7$ y primeras relaciones sexuales $15.3 \pm 2$ años, con niveles de escolaridad predominantes secundario (30\%) y preuniversitario $(30 \%)$. El conocimiento grupal de flujo vaginal pre-intervención fue evaluado de mal $(n=17 ; 73.9 \%)$ con una mediana de 46.00 puntos, en tanto en el periodo post-intervención paso a evaluarse de bien ( $n=12 ; 52.2 \%)$ con una mediana de calificación de 78.00 puntos. En resumen se observó una variación por coeficiente en la calificación pre- pos intervención de 37.43 a 12.74 puntos (TABLA 1). 
TABLA 1. Grado de conocimiento global del flujo vaginal. Las Tunas, 2018

\begin{tabular}{|c|c|c|c|c|c|c|c|c|}
\hline \multirow{2}{*}{$\begin{array}{c}\text { Grado de } \\
\text { conocimiento }\end{array}$} & \multicolumn{5}{|c|}{ Antes } & \multicolumn{4}{c|}{ Después } \\
\cline { 2 - 10 } & Frec & Mediana* & D. E. & C.V. & Frec & Mediana & D. E. & C.V. \\
\hline Muy bien & - & - & - & - & 4 & 95.00 & 1.71 & 1.79 \\
\hline Bien & 3 & 78.00 & 1.15 & 1.49 & 12 & 78.00 & 5.03 & 6.19 \\
\hline Regular & 3 & 67.00 & 1.15 & 1.74 & 7 & 68.14 & 2.85 & 4.19 \\
\hline Mal & 17 & 38.00 & 12.46 & 31.19 & - & - & - & - \\
\hline Total & 23 & 48.00 & 18.07 & 37.43 & 23 & 78.00 & 10.15 & 12.74 \\
\hline
\end{tabular}

En la etapa pre-intervención solo el coito desprotegido fue reconocido como factor en un $(\mathrm{n}=17 ; 73.9 \%)$ de las mujeres, el resto de los riesgos cifró poco significativo lo cual demuestra notable desconocimiento general acerca del tema.

Al comparar las proporciones pre y postintervención, se halló que el incremento en los conocimiento acerca de los factores predisponentes al flujo vaginal fue estadísticamente significativo para una $(p=0.029)$ (TABLA 2).

TABLA 2. Evaluación del conocimiento de factores de riesgo asociados al flujo vaginal. Las Tunas, 2018

\begin{tabular}{|c|c|c|c|c|}
\hline \multirow{2}{*}{$\begin{array}{l}\text { Factores } \\
\text { predisponentes } \mathrm{n}(23)\end{array}$} & \multicolumn{2}{|c|}{ Antes } & \multicolumn{2}{|c|}{ Después } \\
\hline & Frec. & $\%$ & Frec & $\%$ \\
\hline Abuso de antibióticos & 7 & 30.4 & 17 & 73.9 \\
\hline Rasurado Vaginal & 2 & 8.7 & 13 & 56.5 \\
\hline Incorrecto aseo genital & 4 & 17.4 & 16 & 69.6 \\
\hline Coito desprotegido & 17 & 73.9 & 22 & 95.7 \\
\hline Uso de ropa sintética & 9 & 39.1 & 18 & 78.3 \\
\hline Coito Inverso & 11 & 47.8 & 18 & 78.3 \\
\hline \multicolumn{5}{|l|}{ Wilcoxon: p 0.029} \\
\hline \multicolumn{5}{|c|}{$\begin{array}{l}\text { Fuente: elaboración propia con información de la } \\
\text { encuesta. }\end{array}$} \\
\hline
\end{tabular}

El conocimiento acerca de las consecuencias del flujo vaginal se plasmó en la (TABLA 3). En la etapa pre-intervención una media de 6.0 (D.E.3.6) pacientes reconocieron correctamente los indicadores evaluados como consecuencias del flujo vaginal; sin embargo luego de la intervención el aumento del dominio del tema tuvo una media de 19.7 (D.E. 2.9) con una significación estadística de $\mathrm{p}=0.014$.

\begin{tabular}{|c|c|c|c|c|}
\hline \multirow[t]{2}{*}{ Consecuencias n(23) } & \multicolumn{2}{|c|}{ Antes } & \multicolumn{2}{|c|}{ Después } \\
\hline & Frec. & $\%$ & Frec. & $\%$ \\
\hline Infertilidad femenina & 12 & 52.17 & 18 & 78.26 \\
\hline $\begin{array}{l}\text { Infecciones } \\
\text { neonatales y } \\
\text { maternas }\end{array}$ & 7 & 30.43 & 16 & 69.57 \\
\hline Bajo peso al nacer & 2 & 8.70 & 17 & 73.91 \\
\hline Inflamación pélvica & 5 & 21.74 & 21 & 91.30 \\
\hline Parto prematuro & 3 & 13.04 & 23 & 100.00 \\
\hline $\begin{array}{l}\text { Presión de la pareja } \\
\text { como forma de violación } \\
\text { de los derechos } \\
\text { sexuales }\end{array}$ & 5 & 21.74 & 22 & 95.65 \\
\hline Coito doloroso & 9 & 39.13 & 21 & 91.30 \\
\hline \multicolumn{5}{|l|}{ Wilcoxon: $p=0.014$} \\
\hline $\begin{array}{l}\text { Fuente: elaboración prc } \\
\text { encuesta }\end{array}$ & cor & orm & $n d e$ & \\
\hline
\end{tabular}




\section{DISCUSION}

En la literatura científica, las intervenciones acerca de infecciones de transmisión sexual son eficaces al modificar hábitos de higiene y conducta sexual. Reportes de investigaciones abordan los conocimientos en temas relacionados a la sexualidad, infecciones de transmisión sexual e infecciones vaginales. $(18,19,20,6,21)$ Señalan además que las mujeres en edad reproductiva presentan mayor frecuencia flujo vaginal en edades que median los 28 años, datos que concuerdan con los del presente estudio. $(8,22,23)$

La edad de comienzo de las relaciones sexuales concuerdan con los datos del estudio y los presentados por Puentes Rizo (24), Alpízar Navarro (25) y Vidal Borrás (16) quienes plantean que el promedio se encuentra ente los 15 a 17 años, datos similares se muestra en Costa Rica mediante la II encuesta Nacional sobre Salud Sexual y Reproductiva. (26)

El nivel de desconocimiento acerca del flujo vaginal en la etapa pre-interventiva supera los resultados encontrados por Díaz Ortiz, (27) Milán González, (28)Talavera Tamayo (6) y Chacón O'Farrill. (29) El rechazo u obligación al coito por parte de los compañeros sexuales no era vista como una forma de vulneración de los derechos sexuales, concordando con Moreaux Herrera. (30).

El rasurado y la técnica de aseo vaginal incorrecta fueron los riesgos menos asociados a la presencia de flujo vaginal en la etapa inicial al igual que las investigación realizadas por Puentes Rizo, (24) Espinosa Nieto (31) y Álvarez Rodríguez. (32).

El análisis por método estadístico en la presente investigación mostro ascenso de las medidas de tendencia central, disminución de la dispersión y asociación inferencial por la Prueba S-Wilks $(p=0.020)$ lo que induce determinar de efectivo la asimilación del contenido impartido de la intervención educativa.

\section{CONCLUSIONES}

En mujeres en edad fértil existe un inicio precoz de las relaciones sexuales. Se asocia la la presencia de flujo vaginal por desconocimiento de los factores de riesgo y su repercusión en la salud sexual y reproductiva. La intervención educativa basada en la participación contribuye a la adquisición de nuevos conocimientos.

\section{REFERENCIAS}

1. Castillo Pacheco MC. Factores de riesgo de recidivas de infecciones vaginales en mujeres en edad fertil que acuden al susbcentro de salud La Propicia. Ecuador: Pontificia Universidad Católica del Ecuador, Escuela de Enfermería; 2015. Disponible en: https://repositorio.pucese.edu.ec/handle/123456789/402

2. Alemán Mondeja LD, Almanza Martínez C, Fernández Limia O. Diagnóstico y prevalencia de infecciones vaginales. Revista Cubana de Obstetricia y Ginecología. 2010 Junio;36(2):62-103. Disponible en:http://scielo.sld.cu/scielo.php?script=sci arttext\&pid=S0138-600X2010000200008\&lng=es

3. Amabebe E, Anumba DOC. The Vaginal Microenvironment: The Physiologic Role of Lactobacilli. Frontiers in Medicine. 201806 13;5. https://doi.org/10.3389/fmed.2018.00181

4. Mora Agüero SDLÁ. Microbiota y disbiosis vaginal. Revista Medica Sinergia. 2019 01 01;4(1):313. https://doi.org/10.31434/rms.v4i1.165

5. Rigol Ricardo O. Obstetricia y Ginecología. Tercera ed. La Habana: Ciencias Médicas; 2014.

6. Talavera Tamayo M, Pérez Mompié D, Izaguirre Araujo D. Intervención Educativa para disminuir infección vaginal en las gestantes del consultorio No. 16. Ministerio de Salud Pública. [Online].; 2016 [cited 2019 
Febrero 9]. Available

from: http://www.enfermeria2017.sld.cu/index.php/enfermeria/2017/paper/view/397/389

7. Perea EJ. Infecciones del aparato genital femenino: vaginitis, vaginosis y cervicitis. Medicine. [Online].; 2010 [cited $2018 \quad$ Febrero 1]. Available from: http://www.facmed.unam.mx/deptos/microbiologia/pdf/Vaginitis vaginosis cervicitis Medicine2010.pdf

8. Saucedo MA, Cusit YG, Sánchez LI. Factores asociados a la presencia de flujo vaginal en mujeres asistidas en el CAPS № 8 de la ciudad de Corrientes en el periodo 2011-2012. Revista de la Facultad de Medicina de la UNNE. 2015; 35(1): 15-19. Disponible en: http://pesquisa.bvsalud.org/portal/resource/pt/lil$\underline{773210 \text { ?lang=fr }}$

9. Llanes Delgado L, Romay Buchanán M, Gonzalez Cubela AL, Jimenez Lorenzo F. Intervención educativa sobre síndrome de flujo vaginal en un grupo de mujeres en edad fértil. Revista Medicentro. 2011; 15(1): 46-52. Disponible en: http://www.medicentro.sld.cu/index.php/medicentro/article/view/73/102

10. Kenyon C, Colebunders R, Crucitti T. The global epidemiology of bacterial vaginosis: a systematic review. American Journal of Obstetrics and Gynecology. 2013 Dec;209(6):505523.https://doi.org/10.1016/j.ajog.2013.05.006

11. Sobel JD. Vaginitis de la adulta. Clínicas de Ginecología y Obstetricia. Temas Actuales. 4 ed.; 1990.

12. Sweet R, Gibbs R. Infectious Diseases of the Female Genital Tract. Baltimore: Lippincott Williams \& Wilkins; 1991.

13. Simon C. Introduction: Do microbes in the female reproductive function matter?. Fertility and Sterility. 2018 08;110(3):325-326. https://doi.org/10.1016/i.fertnstert.2018.06.041

14. Maria C, Zahra R, Sara P. Prevalence of Cervical-Vaginal Infections in the Pap-Smear Samples in Iran. Global Journal of Health Science. 2013 Dec 19;6(1). https://doi.org/10.5539/gihs.v6n1p201

15. Estruch Rancaño, L; Santín Peña, M; Lantero Abreu, M; Ochoa Soto, R; Joanes Fiol, J; K, Alé Rodiguez. Informe nacional sobre los progresos realizados en la aplicación del UNGASS. [Online]. La Habana: Editorial Lazo Adentro; 2010 [cited 2019 Febrero 9]. Available from: http://data.unaids.org/pub/report/2010/cuba 2010 country progress report es.pdf

16. Vidal Borrás, Hernández González B. Conductas sexuales de riesgo asociadas a las infecciones de transmisión sexual en adolescentes de una comunidad. Revista Habanera de Ciencias Médicas. 2017; 16(4): 625-634. Disponible en: http://scielo.sld.cu/scielo. $p h p ?$ ?script=sci arttext\&pid=S1729519X2017000400014\&lng=es

17. Abril Matute P, Ñauta Baculima J, Arévalo Peláez E. Infecciones vaginales y factores de riesgo relacionados en pacientes atendidas en el área de ginecología del centro de especialidades central $\begin{array}{lllll}\text { cuenca } & \text { (Tesis } & \text { Mestría). } & \text { Ecuador; }\end{array}$ en: http://dspace.ucuenca.edu.ec/bitstream/123456789/28462/1/Tesis.pdf

18. Muñoz Callol JL, Pérez Peña M, Rodriguez Pena Y, Arevalo Turruelles E. Efectividad de una intervención educativa en conocimientos sobre sexualidad responsable en mujeres con discapacidad intelectual. Revista Cubana de Enfermería. 2016; 32(2): p. 171-181. Disponible en: http://scielo.sld.cu/scielo.php?script=sci arttext\&pid=S0864-03192016000200004\&lng=es

19. Llanes Torres H M, Garcia Canosa D, Yedra Sanchez MY. Intervención educativa sobre infecciones de trasmisión sexual. Revista Cubana Medicina General Integral. 2016; 32(4): 1-9. Disponible en:http://scielo.sld.cu/scielo.php?script=sci arttext\&pid=S0864-21252016000400008\&lng=es 
20. Hernández Núñez J, Valdés Yong M, Colque Delgado VS, Roque Arias SM. Síndrome de flujo vaginal en embarazadas de Santa Cruz del Norte. MediMay. 2016; 23(1). Disponible en:http://revcmhabana.sld.cu/index.php/rcmh/article/view/928

21. Olivera Carmenate C. Estrategia educativa sobre sexualidad sana en adolescentes. Revistas Humanidades Médicas. 2014; 14(3). Disponible en: http://scielo.sld.cu/scielo.php?script=sci arttext\&pid=S1727-81202014000300005\&lng=es

22. Valdeiglesias Cabrera N, Medrano Vasquez AO. VAGINITIS EN MUJERES SEXUALMENTE ACTIVAS. SITUA. 2001;10(19).

en: htpp://sisbib.unmsm.edu.pe/bvrevistas/situa/2001 n19/vaginitis mujeres.htm

Disponible

23. Ciudad Reynaud A. Infección vaginal por cándida: diagnóstico y tratamiento. Revista Peruana $\begin{array}{lllll}\text { Ginecología y } \quad \text { Obstetricia. 2007; 53(3): 159-166. Disponible } & \end{array}$ en:http://sisbib.unmsm.edu.pe/bvrevistas/ginecologia/vol53 n3/pdf/a04v53n3.pdf

24. Puentes Rizo EM, Bárbara ED, Jiménez Chacon MC, López Rodríguez P. Comportamiento del Síndrome de flujo vaginal en el Consultorio 16, Policlínico Párraga. Revista Cubana de Obstetricia y Ginecología. 2009; 35(3). Disponible en: http://scielo.sld.cu/scielo.php?script=sci arttext\&pid=S0138$\underline{600 \times 2009000300007}$

25. Alpízar Navarro J, Rodríguez Jiménez P, Cañete Villafranca R. Intervención educativa sobre educación sexual en adolescentes de una escuela secundaria básica. Unión de Reyes, Matanzas, Cuba. Revista Médica Electrónica. 2014; 36(5) ): 572-582. Disponible en: http://scielo.sld.cu/scielo.php?script=sci arttext\&pid=S1684-18242014000500005\&lng=es

26. Dirección de Planificación y Evaluación de Acciones en Salud, Centro Centroamericano de Población, Dirección de Vigilancia de la Salud. II Encuesta Nacional de Salud Sexual y Reproductiva. [Online]. Ministerio de Salud, Universidad de Costa Rica. 2016 [cited 2019 Enero 22]. Available from: https://ccp.ucr.ac.cr/documentos/portal/Informe-2daEncuesta-2015.pdf

27. Díaz Ortiz Y. Intervención educativa en gestantes con infección vaginal [Tesis]. Camagüey: Instituto Superior de Ciencias Médicas Carlos J. Finlay; 2014.

28. Milián Gonzáles I, Martínez Torres YM, Moreno González, Silot Guilbeaux D, Blet Campos R. Intervención educativa para incrementar el nivel de conocimientos sobre infección vaginal en gestantes. $\begin{array}{lllll}\text { Revista Información } & \text { Científica. } & \text { 2018; }\end{array}$ en: http://www.revinfcientifica.sld.cu/index.php/ric/article/view/1887

29. Chacón O'Farrill D, Alfaro C. Intervención educativa del síndrome de flujo vaginal en gestantes. Revista Cubana de Obstetricia y Ginecología. 2018; 44(2). Disponible en:http://www.revginecobstetricia.sld.cu/index.php/gin/article/view/342

30. Moreaux Herrera D, Portuondo Hernández Y, Franco Chibás A, Quiala Portuondo JE, Guilarte Guindo PE. Derechos sexuales en adultos jóvenes. Revista Información Científica. 2016; 95(4): 532-540. Disponible en:http://www.revinfcientifica.sld.cu/index.php/ric/article/view/81

31. Espinosa Nieto CN, Rodríguez Barroso X, Sánchez Quesada S, Alba Martínez D. Intervención educativa a embarazadas con síndrome de flujo vaginal. Revista Digital Buenos Aires. 2015; 19(201). Disponible en:https://www.efdeportes.com/efd201/embarazadas-con-sindrome-de-flujo-vaginal.htm

32. Álvarez Rodríguez JM, Méndez Hernández A, García Verdecia O, Rodríguez Fernández I, Ramos Mustelier F. Epidemiología de la infección vaginal en gestantes diabéticas. MEDISAN. 2014; 18(1): 84-89. Disponible en:http://scielo.sld.cu/scielo.php?script=sci arttext\&pid=S1029-30192014000100012\&lng=es 